\title{
Development of Epstein-Barr Virus-related Primary Diffuse Large B-cell Lymphoma of the Central Nervous System in a Patient with Peripheral T-cell Lymphoma, Not Otherwise Specified after Mogamulizumab Treatment
}

\author{
Hiroaki Tanaka ${ }^{1}$, Hanako Aoki ${ }^{2}$, Yasumasa Sugita ${ }^{1}$, Ryo Shimizu $^{1}$, Katsunari Kiko ${ }^{3}$, \\ Hidetoshi Mochida ${ }^{3}$ and Yoshio Suzuki ${ }^{4}$
}

\begin{abstract}
:
Mogamulizumab is a defucosylated humanized anti-CC chemokine receptor type 4 (CCR4) antibody that exerts an anti-tumor immune effect against various tumors through a suppressive effect on regulatory T-cells. We herein report a patient with peripheral T-cell lymphoma who developed Epstein-Barr virus (EBV)-related primary diffuse large B-cell lymphoma of the central nervous system (CNS DLBCL) after mogamulizumab therapy. Our experience should alert physicians to the possibility of the development of EBV-related CNS DLBCL in patients treated for primary lymphoma and suggests that the anti-tumor immune effect of mogamulizumab is ineffective for the prophylaxis of EBV-related lymphomas.
\end{abstract}

Key words: mogamulizumab, CNS DLBCL, PTCL-NOS, EBV, secondary malignancy

(Intern Med 56: 2759-2763, 2017)

(DOI: 10.2169/internalmedicine.8781-16)

\section{Introduction}

Mogamulizumab is a defucosylated humanized anti-CC chemokine receptor type 4 (CCR4) monoclonal antibody engineered to exert potent antibody-dependent cellular cytotoxicity. Mogamulizumab induces clinically significant antitumor activity in patients with CCR4-positive T-cell lymphomas, such as adult T-cell lymphoma (ATL), peripheral T-cell lymphoma, and cutaneous T-cell lymphoma $(1,2)$. Regulatory T-cells (Tregs) express CCR4, which mediates the homeostatic peripheral tolerance by suppressing autoreactive $\mathrm{T}$ cells. Certain cancers avoid the immune response of the host by recruiting Tregs to the tumor mass (3). Apart from directly killing CCR4-expressing tumor cells, mogamulizumab increases the anti-tumor immune effect of the host response for various tumors by exerting a suppressive effect on Tregs (4).

We herein report our experience with the case of a patient with peripheral T-cell lymphoma, not otherwise specified
(PTCL-NOS) who developed Epstein-Barr virus (EBV)related primary diffuse large B-cell lymphoma of the central nervous system (CNS DLBCL) after undergoing mogamulizumab therapy.

\section{Case Report}

In February 2016, a 66-year-old Japanese man suffered from continuous loss of appetite and fatigue 3 weeks after contracting a cough accompanied by nasal discharge. He was diagnosed with severe anemia and referred to our hospital. The laboratory findings were as follows: white blood cell count, $22.4 \times 10^{9} / \mathrm{L}$ without abnormal cells; red blood cell count, $880 \times 10^{9} / \mathrm{L}$; hemoglobin, $3.1 \mathrm{~g} / \mathrm{dL}$; platelet count, 364 $\times 10^{9} / \mathrm{L}$; reticulocyte count, $13 \times 10^{9} / \mathrm{L}$; lactate dehydrogenase (LDH), $630 \mathrm{U} / \mathrm{L}$ (normal range, 124-226 IU/L); soluble interleukin-2 receptor (sIL-2R), 5,270 IU/mL; C-reactive protein, $2.25 \mathrm{mg} / \mathrm{dL}$; IgG, $1,659 \mathrm{mg} / \mathrm{dL} ; \mathrm{IgA}, 468 \mathrm{mg} / \mathrm{dL}$; and $\operatorname{IgM}, 545 \mathrm{mg} / \mathrm{dL}$. An electrophoretic analysis of proteins revealed the absence of M-protein. A polyspecific direct an-

${ }^{1}$ Department of Hematology, Asahi General Hospital, Japan, ${ }^{2}$ Department of Internal Medicine, Asahi General Hospital, Japan, ${ }^{3}$ Department of Neurosurgery, Asahi General Hospital, Japan and ${ }^{4}$ Department of Diagnostic Pathology, Asahi General Hospital, Japan Received: December 27, 2016; Accepted: March 1, 2017; Advance Publication by J-STAGE: September 15, 2017 Correspondence to Dr. Hiroaki Tanaka, htanaka@ hospital.asahi.chiba.jp 


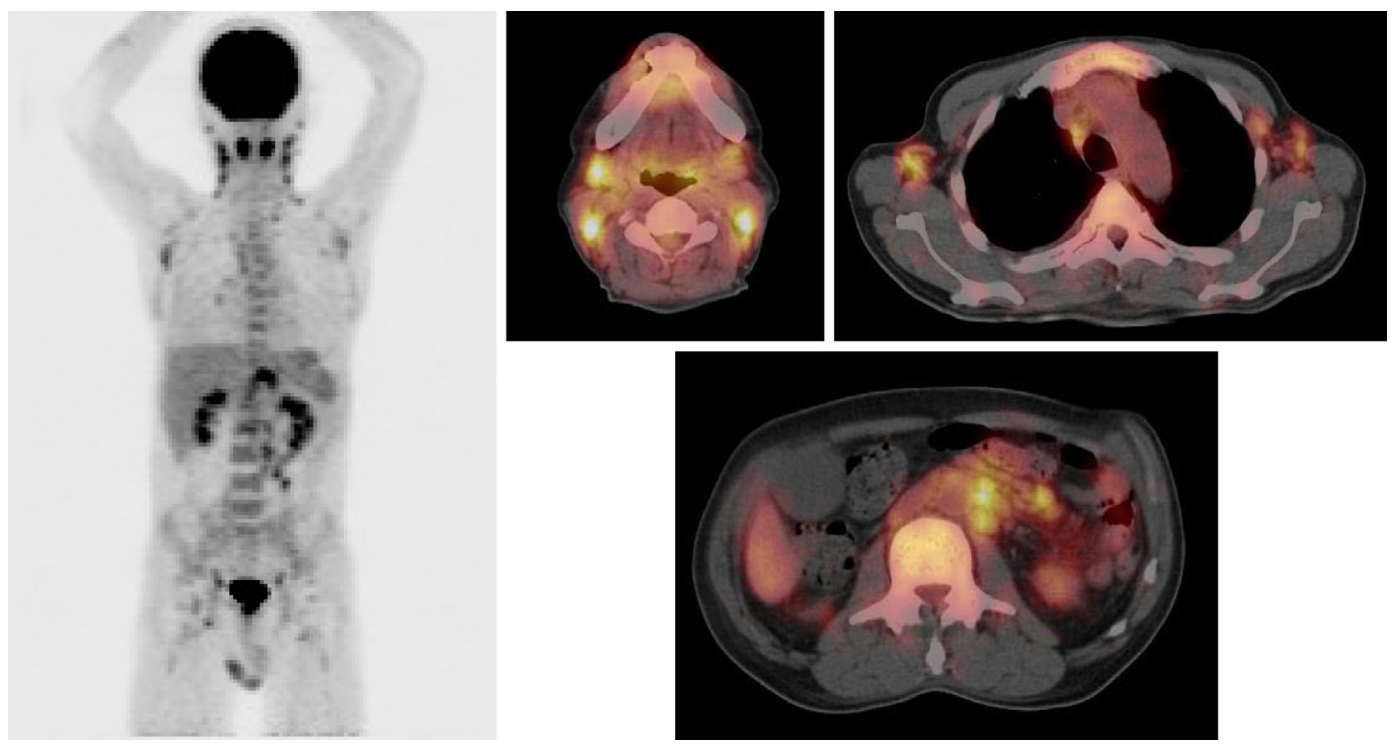

Figure 1. $\left[{ }^{18} \mathbf{F}\right]$-fluorodeoxyglucose positron emission tomography-computed tomography showed abnormal accumulations in the bilateral tonsils and cervical, supraclavicular fossa, axilla, mediastinum, para-aortic, mesenteric, and inguinal lymph nodes.

tiglobulin test (DAT) and a monospecific DAT for $\operatorname{IgG}$ and complement $\mathrm{C} 3 \mathrm{~d}$ were positive. The cold agglutinin titer was 128. Irregular antibody test revealed the presence of anti-E antibodies. Serological tests for human immunodeficiency virus and human T-cell lymphotropic virus type I were negative, and the test for the detection of antimycoplasma IgM was positive. Enhanced computed tomography (CT) revealed multiple lymphadenopathies in the cervical, chest, and abdominal regions. Positron emission tomography detected abnormal accumulations of $\left[{ }^{18} \mathrm{~F}\right]-$ fluorodeoxyglucose in the bilateral tonsils and in the cervical, supraclavicular fossa, axillary, mediastinal, para-aortic, mesenteric, and inguinal lymph nodes (Fig. 1), along with diffuse and high accumulations in the bone marrow.

Transfusion therapy, the avoidance of exposure to cold temperatures, and the administration of $100 \mathrm{mg} /$ body of prednisolone without any other immunosuppressive agent for 3 days were implemented, assuming the patient had secondary cold agglutinin disease due to mycoplasma infection. However, four weeks after the initiation of these treatments, abnormal lymphocytes were detected in the peripheral blood. Flow cytometry revealed that the abnormal lymphocytes to be positive for CD4 and negative for CD8, CD25, and CD56. T-cell receptor gene rearrangement was positive. A bone marrow biopsy revealed proliferation of CD3positive abnormal lymphocytes. An axial lymph node biopsy revealed ambiguous follicles and proliferative abnormal lymphocytes that were positive for CD3, CD4, CD5, and CCR4, as detected by immunostaining (Fig. 2). There was no proliferation of high endothelial venules or follicular dendritic cells. CCR4-positive PTCL-NOS was diagnosed. CHOP therapy $\left(50 \mathrm{mg} / \mathrm{m}^{2}\right.$ doxorubicin, $1.4 \mathrm{mg} / \mathrm{m}^{2}$ vincristine, and $750 \mathrm{mg} / \mathrm{m}^{2}$ cyclophosphamide on day $1 ; 60 \mathrm{mg} / \mathrm{m}^{2}$ prednisone on days $1-5)$ and CHASE therapy $\left(100 \mathrm{mg} / \mathrm{m}^{2}\right.$ etopside on days $1-3 ; 1,200 \mathrm{mg} / \mathrm{m}^{2}$ cyclophosphamide on day 1 ; $1,000 \mathrm{mg} / \mathrm{m}^{2}$ cytarabine on days 2 and 3; and $40 \mathrm{mg} /$ body dexamethasone on days 1-3) were ineffective. Therefore, we next administered $1 \mathrm{mg} / \mathrm{kg}$ of mogamulizumab each week. After seven courses of mogamulizumab therapy, the patient developed right upper limb paralysis. Enhanced brain magnetic resonance imaging revealed multiple brain tumors with ring enhancement (Fig. 3). On a cerebrospinal fluid examination, no abnormal cells were detected; however, EBVDNA was detected. A brain biopsy revealed edema, bleeding, and destruction of the brain parenchyma as well as infiltration by abnormal, small circular cells around the blood vessels. Immunostaining revealed that these abnormal cells expressed L26 but not CD3 and CD5. In situ hybridization revealed that these cells were positive for EBV-encoded small RNA (Fig. 4). These findings led to the diagnosis of EBV-related CNS DLBCL as a secondary malignancy. A review of the findings from the specimen obtained on a previously performed axial lymph node biopsy revealed that the CD20-positive B-lymphocytes around the PTCL cells were also positive for EBV-encoded small RNA. The patient was administered palliative treatment and died while comatose 19 days after admission (4 months after the diagnosis of PTCL-NOS).

\section{Discussion}

Considering the clinical course of the present patient, this brain tumor can easily be misdiagnosed as the progression of PTCL-NOS to the CNS. However, the results of a brain biopsy provided a correct diagnosis of EBV-related CNS DLBCL as a secondary malignancy. Because a brain biopsy is often difficult based on the site, there may be other patients who are incorrectly diagnosed. Physicians should be 


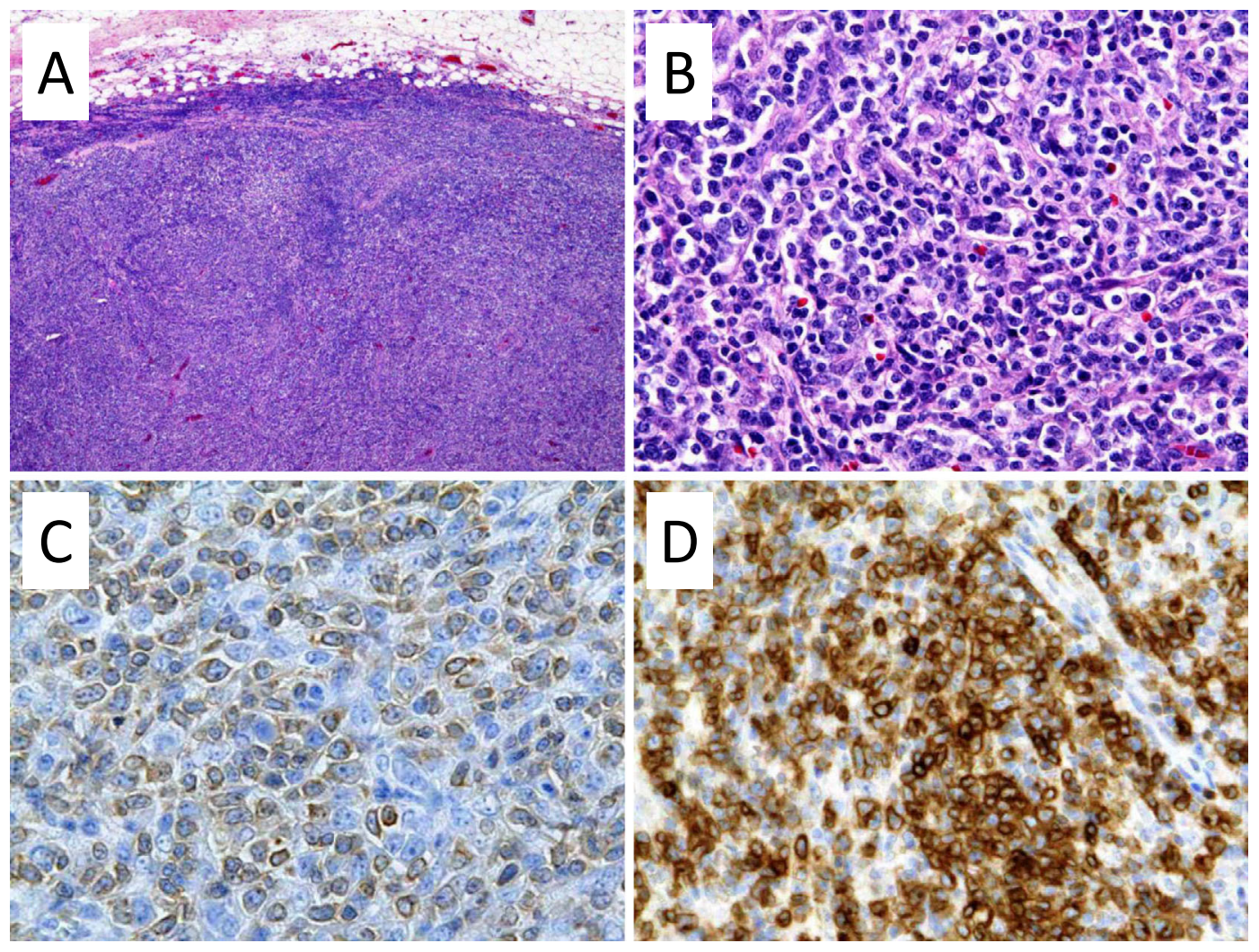

Figure 2. The axial lymph node biopsy specimen revealed ambiguous follicles accompanied by the proliferation of abnormal lymphocytes (Hematoxylin and Eosin staining; A, x100; B, ×400). These lymphocytes were positive for CD3 $(\mathrm{C} \times 400)$ and CCR4 $(\mathrm{D} \times \mathbf{4 0 0})$ by immunostaining.
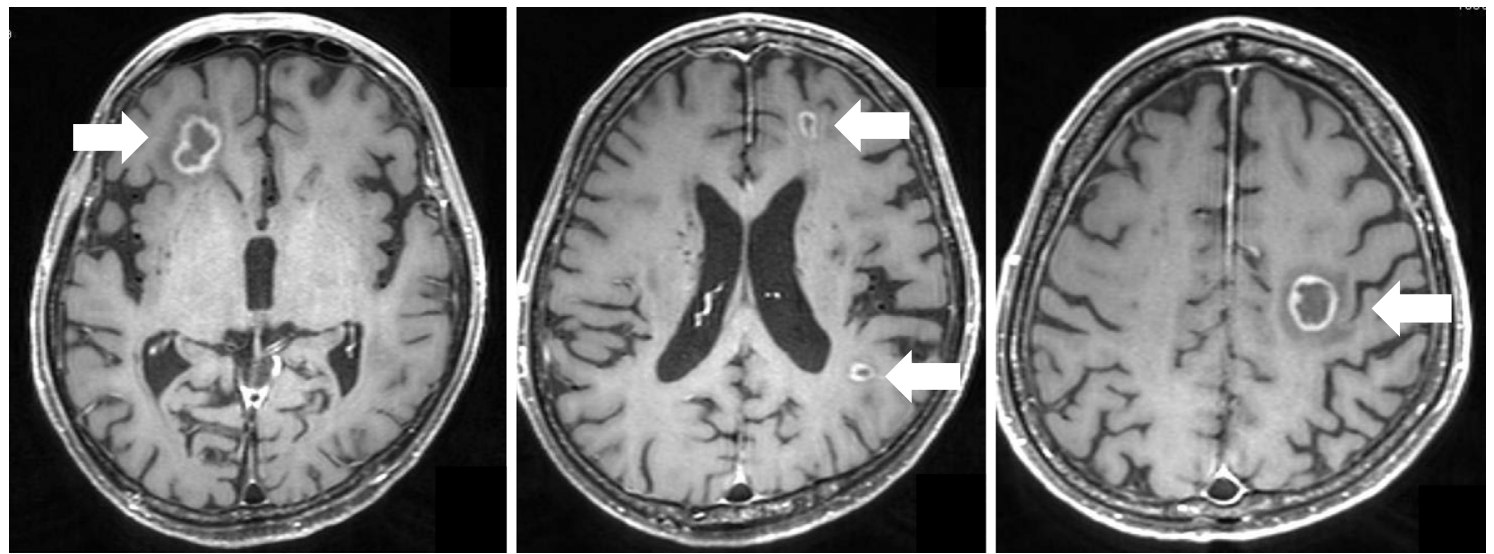

Figure 3. Enhanced magnetic resonance imaging of the brain detected multiple tumors with ring enhancement (arrows).

aware of the possibility of the development of EBV-related CNS DLBCL as a secondary malignancy in patients treated for primary lymphoma.

CNS DLBCL represents all primary intracerebral or intraocular lymphomas, and EBV is generally undetectable in immunocompetent patients with CNS DLBCL $(5,6)$. Most patients with EBV-related CNS DLBCL are immunocompromised because of acquired immune deficiency syndrome or immunosuppression associated with organ transplantation (7). The most significant finding of our present study is that despite the administration of mogamulizumab, which should have increased the host anti-tumor immune effect, secondary malignancy developed. Furthermore, this secondary malignancy was EBV-related CNS DLBCL, which develops in immunocompromised patients. The present case suggests that the anti-tumor immune effect caused by suppressing Tregs may be ineffective for the prophylaxis of EBV-related lymphomas.

There have been some reports describing opportunistic viral infection after mogamulizumab therapy for patients with ATL, such as cytomegalovirus infection $(8,9)$, reactivation of hepatitis B virus (10), and fatal pneumonia and viremia 


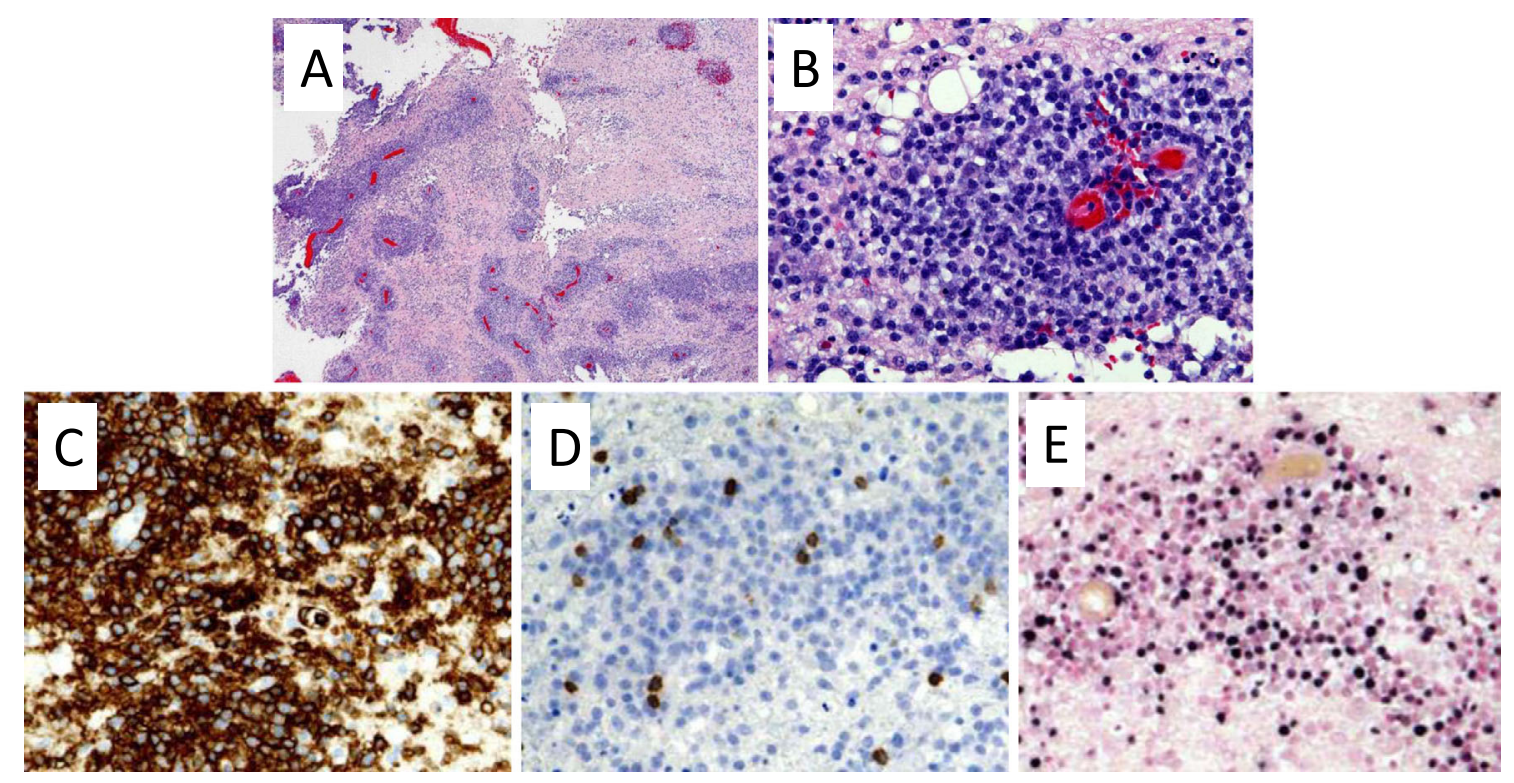

Figure 4. An analysis of the brain biopsy specimens revealed infiltration of abnormal small, circular cells around the blood vessels (Hematoxylin and Eosin staining; A, ×100; B, ×400). Immunostaining revealed that these abnormal cells expressed $\mathrm{L} 26(\mathrm{C} \times 400)$ but not $\mathrm{CD3}(\mathrm{D} \times 400)$. In situ hybridization revealed that these cells were positive for Epstein-Barr virus-encoded small RNA $(\mathrm{E} \times \mathbf{4 0 0})$.

due to parainfluenza virus (11). Indeed, fatal cytomegalovirus pneumonia after mogamulizumab therapy was reported in a patient with T-cell lymphoma other than ATL (12). For the development of tumors with related virus infections, such as that of EBV, the anti-tumor immune effect of mogamulizumab may be ineffective or work in a negative way.

One limitation associated with the present case report warrants mention. We cannot exclude the possibility that severe immunosuppression due to PTCL-NOS itself and the previous chemotherapies already existed before mogamulizumab treatment and resulted in the development of the EBVrelated CNS DLBCL.

In conclusion, we experienced a patient with PTCL-NOS who developed EBV-related CNS DLBCL after undergoing mogamulizumab therapy. Our experience should alert physicians to the possibility of development of EBV-related CNS DLBCL as a secondary malignancy in patients treated for primary lymphoma and suggested that the anti-tumor immune effect caused by suppressing Tregs may be ineffective for the prophylaxis of EBV-related lymphomas. We hope that these findings will help improve our understanding of the anti-tumor immune effect of mogamulizumab on hematological malignancies.

The authors state that they have no Conflict of Interest (COI).

\section{References}

1. Ishida $\mathrm{T}$, Joh $\mathrm{T}$, Uike $\mathrm{N}$, et al. Defucosylated anti-CCR4 monoclonal antibody (KW-0761) for relapsed adult T-cell leukemialymphoma: a multicenter phase II study. J Clin Oncol 30: 837842, 2012.

2. Ogura M, Ishida T, Hatake K, et al. Multicenter phase II study of mogamulizumab (KW-0761), a defucosylated anti-cc chemokine receptor 4 antibody, in patients with relapsed peripheral T-cell lymphoma and cutaneous T-cell lymphoma. J Clin Oncol 32: 1157-1163, 2014.

3. Curiel TJ, Coukos G, Zou L, et al. Specific recruitment of regulatory $\mathrm{T}$ cells in ovarian carcinoma fosters immune privilege and predicts reduced survival. Nat Med 10: 942-949, 2004.

4. Ito A, Ishida T, Yano H, et al. Defucosylated anti-CCR4 monoclonal antibody exercises potent ADCC-mediated antitumor effect in the novel tumor-bearing humanized NOD/Shi-scid, IL-2Rgamma (null) mouse model. Cancer Immunol Immunother 58: 1195-1206, 2009.

5. Mahadevan A, Rao CR, Shanmugham M, Shankar SK. Primary central nervous system diffuse large B-cell lymphoma in the immunocompetent: Immunophenotypic subtypes and Epstein-Barr virus association. J Neurosci Rural Pract 6: 8-14, 2015.

6. Kluin PM, Deckert M, Feery JA. Primary diffuse large B-cell lymphoma of the CNS. In: WHO Classification of Tumours of Haematopoietic and Lymphoid Tissues. 4th ed. Swerdlow SH, Campo E, Harris NL, Eds. IARC, Lyon, 2008: 240-241.

7. Chang KL, Flaris N, Hickey WF, Johnson RM, Meyer JS, Weiss LM. Brain lymphomas of immunocompetent and immunocompromised patients: study of the association with Epstein-Barr virus. Mod Pathol 6: 427-432, 1993.

8. Ishida $T$, Jo T, Takemoto $S$, et al. Dose-intensified chemotherapy alone or in combination with mogamulizumab in newly diagnosed aggressive adult T-cell leukaemia-lymphoma: a randomized phase II study. Br J Haematol 169: 672-682, 2015.

9. Ohyama Y, Kumode T, Eguchi G, et al. Induction of molecular remission by using anti-CC-chemokine receptor 4 (anti-CCR4) antibodies for adult $\mathrm{T}$ cell leukemia: a risk of opportunistic infection after treatment with anti-CCR4 antibodies. Ann Hematol 93: 169$171,2014$.

10. Totani H, Kusumoto S, Ishida $T$, et al. Reactivation of hepatitis B virus (HBV) infection in adult T-cell leukemia-lymphoma patients with resolved HBV infection following systemic chemotherapy. Int J Hematol 101: 398-404, 2015.

11. Tamaki K, Kinjo T, Aoyama H, et al. Fatal pneumonia and viremia due to human parainfluenza virus type 1 in a patient with 
adult T-cell leukemia lymphoma treated with mogamulizumab. J Infect Chemother 21: 820-823, 2015.

12. Ishii $Y$, Itabashi $M$, Numata $M$, et al. Cytomegalovirus pneumonia after anti-CC-chemokine receptor 4 monoclonal antibody (mogamulizumab) therapy in an angioimmunoblastic T-cell lymphoma patient. Intern Med 55: 673-675, 2016.
The Internal Medicine is an Open Access article distributed under the Creative Commons Attribution-NonCommercial-NoDerivatives 4.0 International License. To view the details of this license, please visit (https://creativecommons.org/licenses/ by-nc-nd/4.0/).

(C) 2017 The Japanese Society of Internal Medicine Intern Med 56: 2759-2763, 2017 